\title{
The Study of Euphemism and Its Pragmatic Translation Strategies
}

\author{
Min Luo \\ School of Foreign Languages, Wuhan Polytechnic University, Wuhan, 430028, China
}

Keywords: Euphemism; Cultural differences; Pragmatic translation theory; Pragmatic translation strategies

\begin{abstract}
There is no doubt that euphemism plays an irreplaceable role in people's daily communicative activities. Accordingly, how to fully understand the cultural differences and handle this issue properly has become a research focus of euphemism. so, this paper will lay emphasis on cultural differences and pragmatic translation strategies.
\end{abstract}

\section{Introduction}

With the development of globalization, more emphasis is being placed on the translation studies to achieve efficient communication among people from different countries. One of the most indispensable study among the wide range of translation studies is the study of euphemism. As it is frequently used in English as well as in Chinese languages, euphemism plays a significant part in cross-cultural activities. In order to prob a deeper understanding of euphemism, linguist have offered various definitions to it, most of which adopts the perspective of semantics. However, there is an increasing focus on the application of pragmatics in euphemism translation, given the fact that euphemism is of practical significance in our daily life. More and more scholars have shifted their focus to the study of pragmatic translation strategies of euphemism while studying the cultural differences between English and Chinese cultures.

\section{The Necessity of Euphemism Studies}

Being not only a linguistic and cultural phenomenon, euphemism is also a tool to help people achieve the effective communication. In the era of globalization, the use of euphemism in the whole world will be more and more frequent. However it is inevitable for people to come across obstacles when use euphemism in communication, because euphemism from different cultural backgrounds contains different cultural connotations. As a result, it is particularly important to understand the cultural connotation of euphemism of different countries, and to seek efficient translation strategies so as to realize the cross-cultural communication successfully. Accordingly, the euphemism translation studies should also be approached from perspective of the social characteristics of euphemism. For a rather long time, translation theorists have done a lot of research on the comparison between Chinese and Western euphemism, which have born fruitful results. When it comes to translation studies of the euphemism, however, more attention is paid on the research from the perspective of semantics, so the social contextual factors are ignored. Thereby, the actual operation effect of the translation text is greatly reduced. Studying euphemism translation strategies from the perspective of pragmatics can not only fully express the psychological motivation of the euphemism users, but also keep the translation text in an accurate and appropriate manner, so as to bring the study of euphemism translation into a brand new stage.

\section{The Analysis of Chinese and English Euphemism}

As one of the most important communicative tools in human society, language generates and develops with the development of the times and areas. During this process, language has been inevitably marked the imprint of the society. As is known to all, language and culture are closely interrelated to each other. Just as Claire Kramsch put it briefly and precisely in three sentences: 
language expresses cultural reality. Language embodies cultural reality. Language symbolizes cultural reality. Euphemism functions as a rhetorical device and communicative tool, and is ubiquitous in all kinds of language in the word. Its' development serves as a lubricant in the communicative activities, which brings convenience for social interactions. The application of euphemism means to talk elegantly in the communicative process, that is to say to replace the expression that may cause displease or awkwardness with something nicer and more easily acceptable by others. There is no doubt that language is an vital carrier of culture. Being a special kind of language, euphemism reflects culture in unusual conditions. The differences between Chinese and Western euphemism not only enrich these languages, but provide us a breakthrough point to study the different cultural backgrounds behind the language.

The translation of euphemism is an ineluctable barrier for translators. Translating euphemism requires the translator to get hang of both Chinese and English very well. Furthermore, translation studies of the euphemism requires the translator to be familiar with the cultural background of the source language and the target language to make sure no misunderstandings of cultural connotations will happen. To make a summary, the factor of culture should be paid more attention to, and translation strategies of euphemism should be approached from the perspective of culture.

The main differences between cultural connotations of Chinese and English euphemism can be divided into the following four aspects: Firstly, the use of euphemism reflects different cultural values. Secondly, the use of euphemism reflects different religious beliefs. Thirdly, the use of euphemism reflects different social classes and social ethics. Fourthly, the use of euphemism reflects different ritual culture.

To sum up, although English and Chinese euphemism share a common psychological basis, including taboo psychology, polite psychology, and so on, the cultural connotations of euphemism is various due to the differences between English and Chinese linguistic features, cultural values, social backgrounds, religious backgrounds, and other aspects. Understanding these differences helps us to eliminate obstacles, so as to achieve the goal of mutual understanding and smooth communication in cross-cultural communication activities.

\section{The Pragmatic Translation Strategies of Euphemism Studies}

Language is an indispensable tool for human communication. Different social environment creates different culture, and to a certain extent, culture can determine the language and behavior of people. Therefore, the meaning of language depends on its' specific social and cultural context. At present, the public hold divergent opinions towards the definition and category of pragmatics. However, a common consensus has been reached that the core connotation of pragmatic is context. The most accepted understanding of pragmatics is that it's a branch of linguistics, which mainly studies the dynamic discourse meaning, and focuses on how people achieve their intentions through semantic and syntactic expressions. Pragmatics is, in short, the study of language application and communication.

No exchanges of people can happen without specific contexts, and contexts can exert influence on the understanding and use of language directly. Translation is, in the essence, the process of using target language to express the meaning of source language. So we can arrive at a natural conclusion that translation should be completed in a certain context, and we must apply for pragmatics in the process. Pragmatics plays the role of a bridge in the translation process, as the application of pragmatic theory in translation can make the meaning of the source language and the meaning of the target language more equivalent, so as to achieve the highest standard of translation. To probe further into the studies of euphemism in social communication activities, the pragmatics of euphemism should be related to.

Pragmatic Translation Studies. With the development of pragmatics, some disciplines such as translation studies, applied linguistics have applied pragmatics in their theories. According to pragmatic translation theory, translation is an activity for exchanging information, thus the pragmatic 
meaning in the process of communication should be emphasized, as well as the equivalent pragmatic effects which readers gain from the original text. As pragmatics studies develop, it has exerted more and more influence on translation, the research on pragmatic translation is increasingly drawing the attention of scholars home and abroad. Some of them have formed their own theories, providing a new research angle for the perfection of translation theory.

The domestic research on pragmatic translation can be traced back to Zhao Yuanren, who asserts that words meaning is determined by the semantic contexts. He also emphasizes function equivalence and pragmatic equivalence. Since him, a series of studies began to crop up in the late 80s, which are represented mainly by Zhang Yafei, Zeng Xiancai, He ziran, Qian Guanlian, and so on. Zhang Yafei pointed out that translation should pay attention to the structure equivalence, semantic equivalence and pragmatic equivalence between source language and target language. Zeng Xiancai put forward his own interpretation of pragmatic translation. He believes that the first and foremost task for translation is to reproduce the meaning of the original text. He thinks semantic translation is the translation of semantic meaning and pragmatic meaning. He Ziran considers pragmatic translation as equivalent translation, which can be divided into pragmatic language equivalence translation and social pragmatic equivalence translation. He proposed that translator should pay attention to context of the original text, find relevance between two texts, and bring various pragmatic strategies to flexible use in dealing with cultural differences between source language and target language. Similarly, Qian Guanlian contends the implicit intention of the writer must be retained in translation. So in order to obtain the perfect equivalent translation, creations should be made on the basis of being faithful to the original text, and relying on the specific context. However, these theories have not formed the specific system of pragmatic translation theory.

It is known that the earliest studies on pragmatic translation abroad are represented by Hatim and Mason. They discussed the relations between speech acts, cooperative principles and translation from the perspective of pragmatic translation. However, this theory proved to be in lack of actual translation operation. British linguist Bell approached translation from the perspective of cognition, which is difficult to implement. The relevance theory proposed by Gutt emphasizes the basic guideline of translation is relevance. The translator's responsibility is to balance writers' purposes and readers' expectation. Obviously speaking, his opinion shares many similarities with He Ziran's and Qian Guanlian's. Leohickey put forward in his work pragmatics and translation the importance and effects of pragmatics in translation in achieving readers' equivalent understanding and feelings of the original book and the translated book. Besides what have mentioned above, it is also worth mentioning about the translation studies in Russia. The pragmatic research in Russia mainly discussed the conveyance of pragmatic meaning and the pragmatic meaning of translation. There are three Russian scholars studying in translation theory probed further into this realm:Barkhudarov, Komissarov and ADBeeP. Their main focuses are: pragmatic relationship, the communicative intention of the original text, the conveyance of pragmatic meaning and research types of pragmatic translation and so on. Their research has born fruitful results.

However, it can be seen that, in general, Chinese and Western scholars both failed to form specific concepts of pragmatic translation and operational translation theories. More attention and efforts should be paid on this filed.

Pragmatic Translation Strategies of Euphemism. First of all, something should be explained that, the premise of euphemism translation, or the first step is to distinguish the emotional meaning of euphemism, and to decide whether it is an expression of euphemism or not. As Larson said, the translation of euphemism should apply the corresponding form of expression in the target language. The key to this is that the translator must be able to recognize whether it is euphemism in the source language. Besides that, the translator should choose wisely the expression that is acceptable to the target readers, be it euphemistic, or straightforward. Pragmatic translation theory demonstrates that the translation of euphemism depends on the context, which means that if there is no meaning of euphemism in the original text, and the translator misunderstands it, or makes a mistake on the 
emotional color of euphemism, big mistakes and misunderstandings will happen. For an instance, the sentence 'He died during the WW II ', its intention maybe is to state the simple fact.

Over the recent years, many Chinese scholars has put forward their views on pragmatic translation strategies. They arrived at a conclusion that the core of pragmatic translation is to achieve translation equivalence, which emphasizes on the function equivalence of information and purpose. Translators should not be restrained by the form of the original text, instead, they should seek to retain the original content with the closest and most natural equivalent expression to its content.

In the following part, the translation strategies of euphemism will be discussed along with some specific examples in order to achieve translation equivalence.

Literal Translation. As language, Chinese and English enjoy lots of common euphemistic expressions, especially in certain aspects concerning manners, death, and other social aspects. In cases like these, the equivalent expression can be easily found, so literal translation can be applied. However, more often is the case that, there is no expression in the target language equivalent or similar to the source language. In cases like these, the translation of euphemism should adopt the strategy of literal translation, that is, to adapt to the cultural background of the context, employ the most appropriate expression to the sentence, so as to convey the meaning of the sentence accurately.

Free translation. The application of free translation can be divided into the following two situations:the first situation is that there is a equivalent or rather relative expression in the target language. The second situation is that there is no equivalence, and free translation should be applied in order to achieve the euphemistic effect. The comparative study of the cultural connotations of English and Chinese euphemism conducted above has offered lots of expressions in Chinese and English about'death', so most of the euphemism concerning these topics can be found equivalence or alternative expressions.

Transliteration with annotation. In some cases, in order to achieve the purpose of foreignization, that is to allow readers fully understand the source language, and to maintain the original flavor of the source language, discard foreign flavor, the strategy of transliteration with annotation can be adopted.

\section{Summary}

To make a final conclusion of the whole thesis, in order to achieve equivalence translation of euphemism, translators need to understand the implied meaning of euphemism based on the cultural context and their analysis. To achieve that, translators must know and be familiar with both languages and some cross-cultural knowledge very well. Only in this way, can misunderstandings and language barriers be avoided as much as possible, so as to attain translation equivalence and communicative effect to the highest degree.

\section{References}

[1] Allan. Keith \& Burridge. Kate. Euphemism and Dysphemism[M]. Oxford: Oxford University press, 1991.

[2] Enright, D.J. Fair of Speech: The Use of Euphemism [M]. Oxford: Oxford University Press, 1985.

[3] Gutt E.A. Translation and Relevance: Cognition and Context [M]. Oxford: Blackwell, 1991.

[4] Hatim, Mason. Discourse and the Translator [M]. London and New York: Longman, 1990.

[5] Kramsch, C. Language and Culture [M]. UK: Oxford University Press, 1998. 